\title{
El capital humano como factor determinante en la calidad de las obras
}

\author{
E. L. Córdova Nativí1
}

${ }^{1}$ Fondo de Conservación Vial de El Salvador (FOVIAL), km. 10 1/2 Carretera al Puerto de la Libertad, El Salvador.

Información del artículo

DOI:

http://dx.doi.org/10.21041/ra.v2

$\underline{\text { i1.24 }}$

Artículo recibido el 01 de

Octubre de 2011, revisado bajo

las políticas de publicación de

la Revista ALCONPAT y

aceptado el 12 de Diciembre de

2011. Cualquier discusión,

incluyendo la réplica de los autores se publicará en el tercer número del año 2012 siempre y cuando la información se reciba antes del cierre del segundo número del año2012

(c) 2012 Alconpat Internacional

Información Legal

Revista ALCONPAT, Año 2, No. 1, EneroAbril 2012, es una publicación cuatrimestral de la Asociación Latinoamericana de Control de

Calidad, Patología y Recuperación de la Construcción, Internacional, A.C., Km. 6 , Construcción, Internacional, A.C., Km. 6,
antigua carretera a Progreso, Mérida Yucatán, antigua carretera a Progreso, Mér alconpat.int@gmail.com, Página Web: www.alconpat.org

Editor responsable: Dr. Pedro Castro Borges. Reserva de derechos al uso exclusivo No.042013-011717330300-203, eISSN 2007-6835, ambos otorgas por el Instituto Nacional ambos otorgados por el Instituto Nacional de Derecho de Autor. Responsable de la última Informática ALCONPAT, Ing. Elizabeth Sabido Maldonado, Km. 6, antigua carretera a Progreso, Mérida Yucatán, C.P. 97310 , fecha de publicación: 30 de enero de 2012

Las opiniones expresadas por los autores no necesariamente reflejan la postura del editor. Queda totalmente prohibida la reproducción total o parcial de los contenidos e imágenes de la publicación sin previa autorización de la ALCONPAT Internacional A.C.

\section{RESUMEN}

En el año 2007, con el objetivo de contar con una Unidad que dé seguimiento y asistencia técnica al control y aseguramiento de la calidad de las obras que ejecuta el Fondo de Conservación Vial de El Salvador (FOVIAL) ${ }^{(1)}$, se crea dentro de la institución, la Unidad de Asesoría de la Calidad.

En el 2008, la Unidad de Asesoría de la Calidad, en la búsqueda de la mejora continua, inicia un programa piloto orientado a la actualización de conocimientos que contribuyan a enriquecer el nivel técnico del personal de las empresas supervisoras y contratistas que laboran en proyectos del programa de mantenimiento periódico. Habiéndose obtenido buenos resultados, se extiende a todos los programas de mantenimiento.

En este documento se incluyen detalles del programa de capacitación e investigaciones aplicadas implementado por FOVIAL y los logros obtenidos en la calidad de las obras, a través de la formación técnica del capital humano.

Palabras clave: capital humano; capacitación; investigaciones; calidad.

\section{ABSTRACT}

Quality Assistance Unit is a Technical Department of Fondo de Conservación Vial (FOVIAL- El Salvador), created in 2007 to monitoring and technical attendance to Quality Control and Quality Assurance to all of FOVIAL maintenance projects.

In 2008, according to continuous improvement, Quality Assistance Unit started a pilot program directed toward updating of knowledge, contributing to enrich the technical level of Supervisors and Contractors Companies, who have periodic maintenance contracts of pavement roads. Having obtained very good results in this pilot program, it is decided to extend to all of the FOVIAL projects.

This document includes details of the training program and applied researches carried out by FOVIAL and achievements obtained in quality works, through the technical training of human resource.

Key words: human resource; training; research; quality.

Autor de contacto: Edith L. Córdova Nativí (ecordova@fovial.com) 
Revista ALCONPAT, Volumen 2, Número 1, Enero-Abril 2012, Páginas 29 - 36

\section{INTRODUCCION}

La creciente demanda de proporcionar mantenimiento a la red vial, la necesidad de mejorar el deficiente desempeño y la escases de fondos, hacen evidente la necesidad de mejorar la calidad en la ejecución de las obras y la optimización de recursos.

Habiendo analizado las diferentes causas de las deficiencias en la calidad de las obras, se detectó que el factor humano guarda una relación directa con el desempeño adecuado de la obra ejecutada, y que muchos de los errores cometidos, han sido producto del desconocimiento en temas referentes al control de calidad en la ejecución de las obras. Con la finalidad de disminuir el volumen de obra No Conforme ${ }^{(1)}$, el FOVIAL cuenta a la fecha con un programa de capacitación continua dirigido al personal que labora en los diferentes programas de mantenimiento.

\section{PROGRAMA DE CAPACITACION}

El programa de capacitación implementado por FOVIAL se basa en la identificación y determinación de requerimientos técnicos de los programas de mantenimiento vial que lleva a cabo la institución. A la vez que se considera como una finalidad primordial, brindar a los profesionales que laboran en los diferentes proyectos, los conocimientos técnicos necesarios para la toma de decisiones, que conlleven a la mejora de los procesos constructivos y por consiguiente, el logro de buenos resultados. En este sentido, el programa de capacitación implementado por FOVIAL se fundamenta en tres aspectos básicos: el enfoque de los conocimientos, la metodología de enseñanza-aprendizaje empleada para impartir los conocimientos y la evaluación, los cuales se explican a continuación:

\subsection{Enfoque de conocimientos}

Para formular el enfoque del programa de capacitación, se determinó cuales conocimientos era necesario reforzar, en este sentido, en el segundo semestre del año 2007, aprovechando las inspecciones periódicas realizadas a los proyectos, se realizó un sondeo acerca de las deficiencias ocurridas con mayor frecuencia y sus posibles causas, detectándose las descritas en la tabla 1:

Tabla 1. Deficiencias detectadas en los proyectos de mantenimiento vial (año 2007)

\begin{tabular}{|c|c|}
\hline Deficiencias detectadas. & Causas probables. \\
\hline $\begin{array}{l}\text { Grado de compactación menor al requerido y } \\
\text { acabado deficiente de la carpeta de mezcla asfáltica. } \\
\text { Procesos inadecuados en la construcción de } \\
\text { pavimentos rígidos y ensayos de laboratorio. } \\
\text { Resultados deficientes en los procesos de } \\
\text { estabilización de suelos con cemento y reciclado de } \\
\text { pavimentos flexibles con cemento. } \\
\text { Resultados inadecuados en los procesos de balastado } \\
\text { de las vías. } \\
\begin{array}{l}\text { Deficiencias debidas a materiales fuera de } \\
\text { especificación. }\end{array}\end{array}$ & 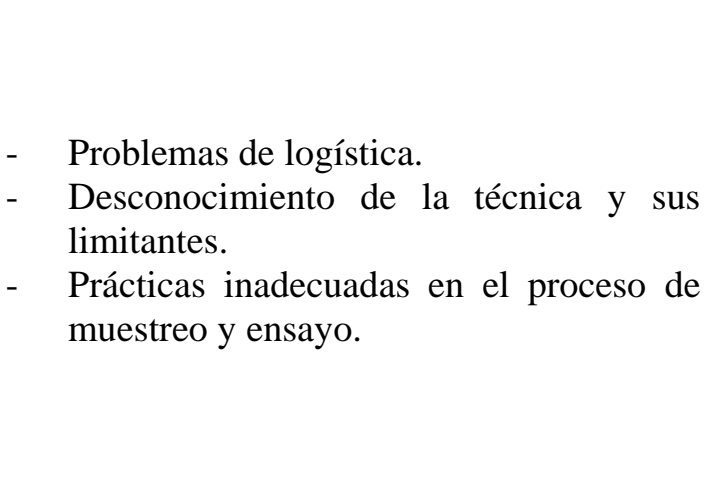 \\
\hline
\end{tabular}

${ }^{(1)}$ Obra No Conforme: producto que no cumple con los requerimientos de calidad especificados para el proyecto. 
Revista ALCONPAT, Volumen 2, Número 1, Enero - Abril 2012, Páginas 29 - 36

Adicionalmente, en el periodo comprendido entre mayo y julio del año 2008, se realizó un análisis estadístico de No Conformidades ${ }^{(2)}$ impuestas a obra que no cumplía con los parámetros de calidad requeridos por FOVIAL, dicho análisis reforzó el sondeo realizado en el año 2007, obteniéndose los siguientes datos:

- De un total de 63 No Conformidades impuestas en este periodo, $36 \%$ de las No Conformidades se daban en la actividad de balastado de vías no pavimentadas y el $33 \%$ correspondía a la actividad de mantenimiento de pavimentos flexibles. Figura 1.

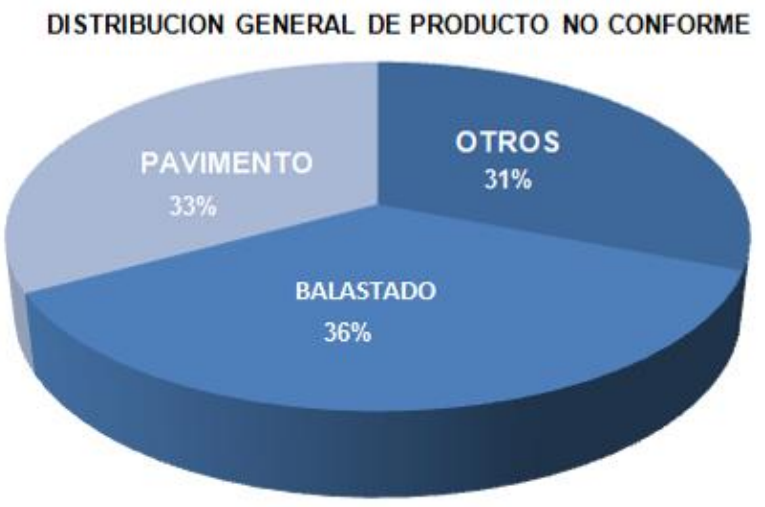

Figura 1. Representación del análisis estadístico general de No Conformidades, vías pavimentadas y vías no pavimentadas

- En el mantenimiento de vías pavimentadas, el $81 \%$ de las No Conformidades eran originadas por proceso constructivo inadecuado y el $19 \%$ eran originadas por materiales que no cumplían con la especificación técnica. Figura 2.

- En el mantenimiento de vías no pavimentadas, el $96 \%$ de las No Conformidades eran originadas por procesos constructivos inadecuados y el $4 \%$ eran originadas por materiales fuera de especificación técnica. Figura 3.

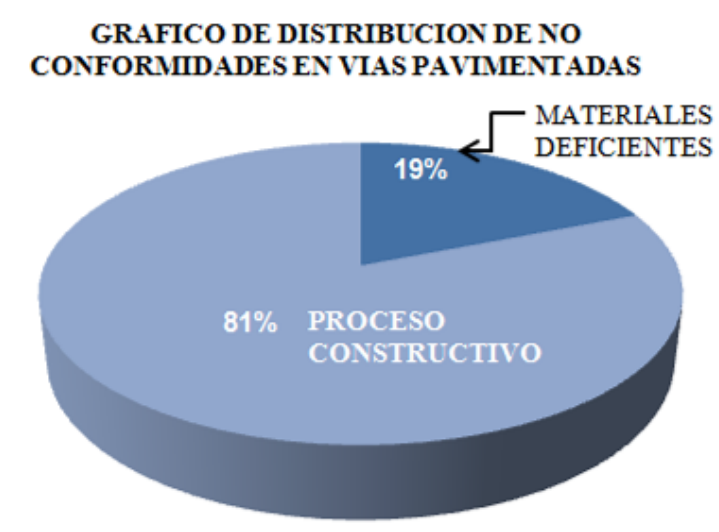

Figura 2. Representación del análisis estadístico de No Conformidades en vías pavimentadas

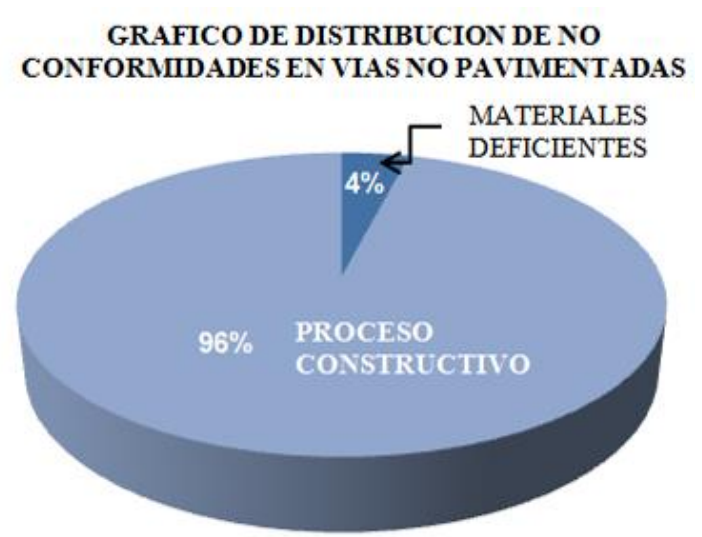

Figura 3. Representación del análisis estadístico de No Conformidades en vías no pavimentadas

\footnotetext{
(2) No Conformidad: estatus de un producto que no cumple con los requerimientos de calidad especificados para el proyecto. Al cual se da el respectivo tratamiento, tomando como referencia la Norma ISO 9001.
} 
Tomando como base la información antes expuesta, FOVIAL dio inicio al programa de capacitación en el año 2008, basándose en la necesidad de disminuir el producto No Conforme, mediante la mejora de la capacidad técnica de los profesionales que laboran en los diferentes programas de mantenimiento vial. Para ello, se plantearon dos líneas de capacitación, las cuales se describen a continuación:

- Capacitaciones sobre la "Implementación del Sistema de Control de Calidad", con lo cual se busca inculcar una cultura de calidad a los profesionales de las empresas contratistas y supervisoras, mediante la implementación del Plan de Control de Calidad y Guía sobre el manejo de la información de control de calidad que se origina en los proyectos.

- Capacitaciones relacionadas con conceptos sobre: diseño, construcción y supervisión de pavimentos rígidos y flexibles, estabilización de suelos utilizando diferentes técnicas, reciclado de pavimentos flexibles, entre otros. Figuras 4 y 5.

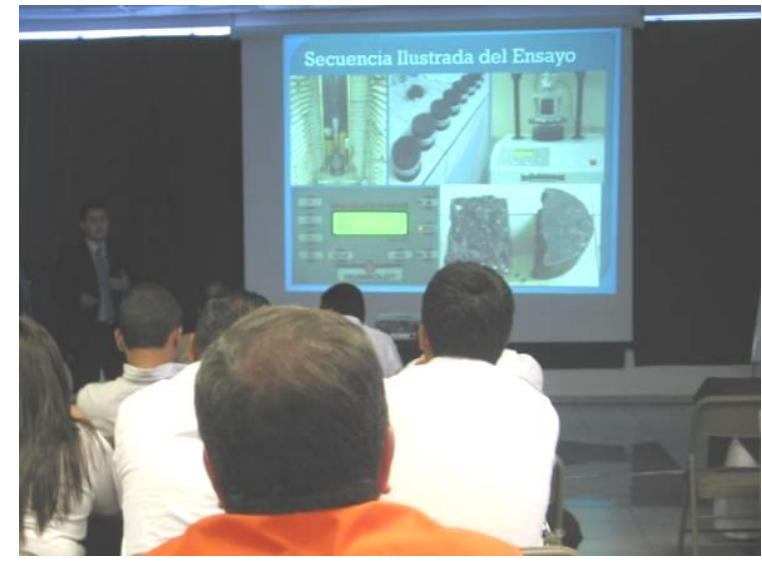

Figura 4. Instante en que se imparten conceptos relacionados con el diseño y construcción de pavimentos flexibles.

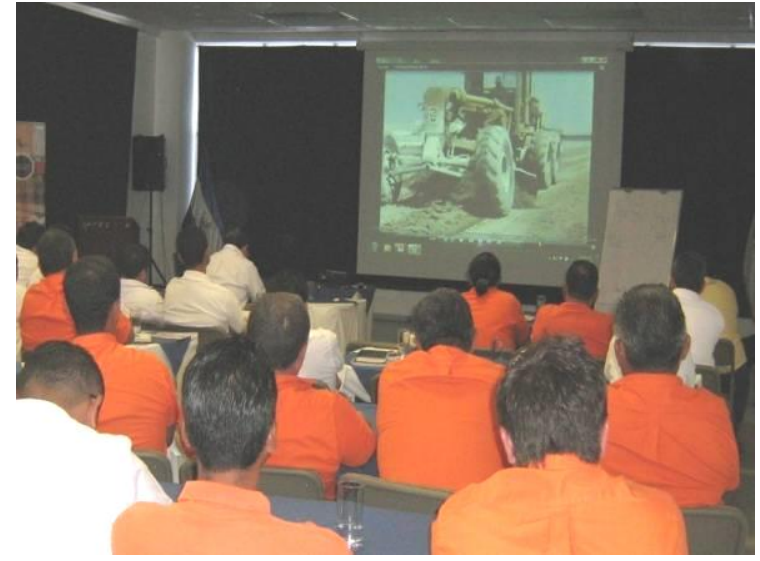

Figura 5. Instante en que se imparten conceptos relacionados con el proceso constructivo de estabilización de suelos.

\subsection{Metodología de enseñanza- aprendizaje}

El proceso de enseñanza-aprendizaje llevado a cabo mediante el programa de capacitación del FOVIAL, consiste en un proceso dinámico, en el cual se asigna al profesional un papel activo, considerándolo sujeto y no objeto del proceso; a fin de estimular al profesional en su proceso de actualización de conocimientos, lo cual conlleva implícitamente a la ejecución de obras con mejor calidad. En definitiva, la metodología del programa de capacitación del FOVIAL se divide en tres momentos básicos, que se describen a continuación:

2.2.1 Facilitación de herramientas para el control de calidad. Estas capacitaciones son facilitadas por personal del área de Asesoría de la Calidad del FOVIAL y su contenido gira alrededor de la siguiente temática:

- Implementación del Plan de Control de Calidad, el cual constituye la herramienta para llevar a cabo el control y el aseguramiento de la calidad de las obras, con el objetivo de guiar al profesional en aspectos relacionados con el manejo de obra No Conforme, implementación de correcciones y acciones preventivas, y seguimiento del proyecto por medio de inspecciones preparatorias, inspecciones iníciales e inspecciones de seguimiento, entre otros.

- Guía sobre el manejo de la información de control de calidad generada en el proyecto, resolución de inquietudes sobre aspectos relacionados con las condiciones técnicas de los proyectos, interpretación de resultados de ensayos realizados a los diferentes materiales 
utilizados, análisis estadístico de resultados de ensayos realizados y presentación de informes técnicos.

2.2.2 Fortalecimiento de conocimientos técnicos. Se desarrolla por medio de charlas técnicas, enfocadas a brindar una actualización de conocimientos relacionados con el diseño, construcción y supervisión de pavimentos rígidos y flexibles, estabilización de suelos, reciclado de pavimentos, entre otros. Durante estas charlas se promueve la participación de los profesionales en etapas demostrativas de ensayos, de procesos constructivos y de preguntas y respuestas, a fin de hacer más dinámico el proceso de enseñanza -aprendizaje, logrando una retroalimentación a todos los interesados. Cabe mencionar, que en este caso se cuenta con la colaboración de entidades expertas en temas relacionados con el mantenimiento vial, con algunas de las cuales FOVIAL ya se ha establecido convenios de colaboración. Figuras 6 y 7.

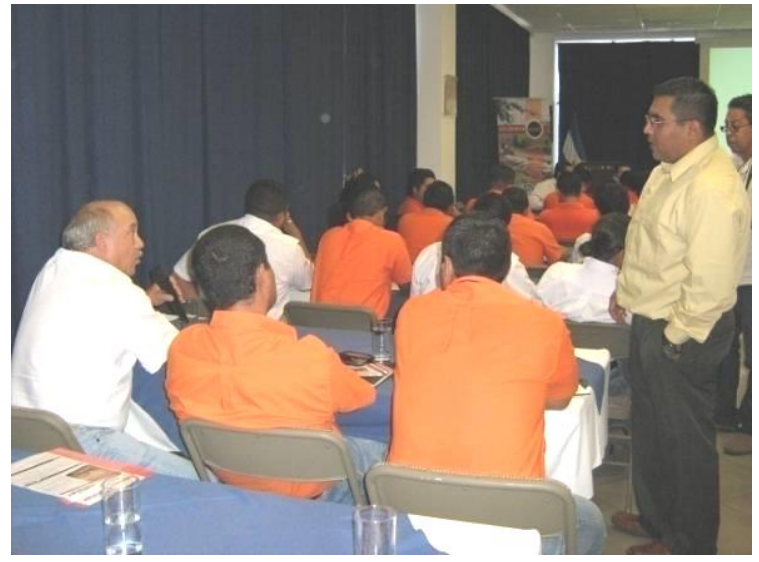

Figura 6. Etapa de preguntas y respuestas durante charla técnica.

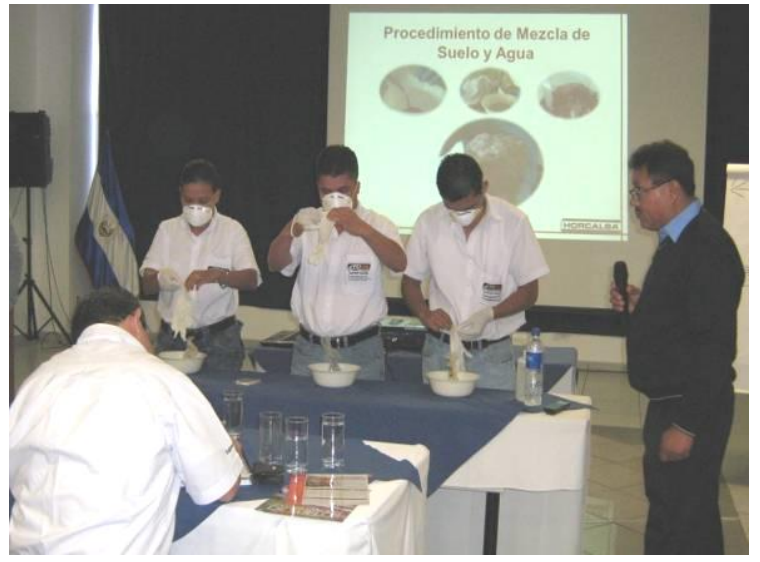

Figura 7. Demostración práctica realizada durante charla técnica.

2.2.3 Investigaciones aplicadas. En esta etapa, la teoría es puesta en práctica, mediante la construcción de tramos de prueba aplicando diferentes técnicas tradicionales y no tradicionales en nuestro país, que permitan atender de manera óptima las necesidades de mantenimiento de la red vial, tales como: estabilización de suelos, reciclado de pavimentos flexibles y tratamientos superficiales.

El proceso de investigación aplicada inicia con la elección del sitio a intervenir, continua con el muestreo y ensayo de materiales, para la elaboración de diseños de mezclas, construcción del tramo de prueba y su posterior seguimiento de desempeño. Como puede observarse, para llevar a cabo esta etapa del proceso de enseñanza-aprendizaje, además de contar con los conocimientos técnicos necesarios, se requiere una adecuada coordinación entre los profesionales y personal de campo de contratista, supervisor, entidades expertas en el tema y personal técnico de FOVIAL, y en algunos casos con entidades donantes.

Un ejemplo del proceso de investigación aplicada, ejecutada por FOVIAL, es el realizado en Suchitoto, Departamento de Cuscatlán, donde unieron esfuerzos personal técnico de FOVIAL, personal de las empresas constructora y supervisora, entidades expertas en el tema de estabilización de suelos compresibles y entidades donantes de cementantes, para dar inicio a la investigación sobre el desempeño de tramos de prueba construidos en vías no pavimentadas, en los cuales se estabilizaron suelos con características plásticas por medio de técnicas, tales como: 
Pavimentos Unicapa de Alto Desempeño (PUAD) ${ }^{(3)}$, Estabilización con Cal, Estabilización con Cal y Cemento, Estabilización con Emulsión Asfáltica y Estabilización Mecánica con Balastado. Figuras 8 y 9.

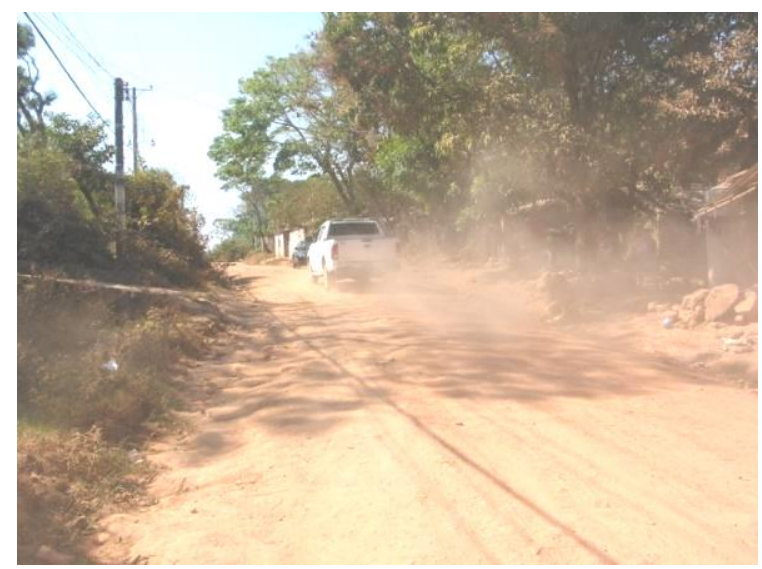

Figura 8. Tramo de vía antes de la construcción de un tramo de prueba de estabilización de suelo con características plásticas.

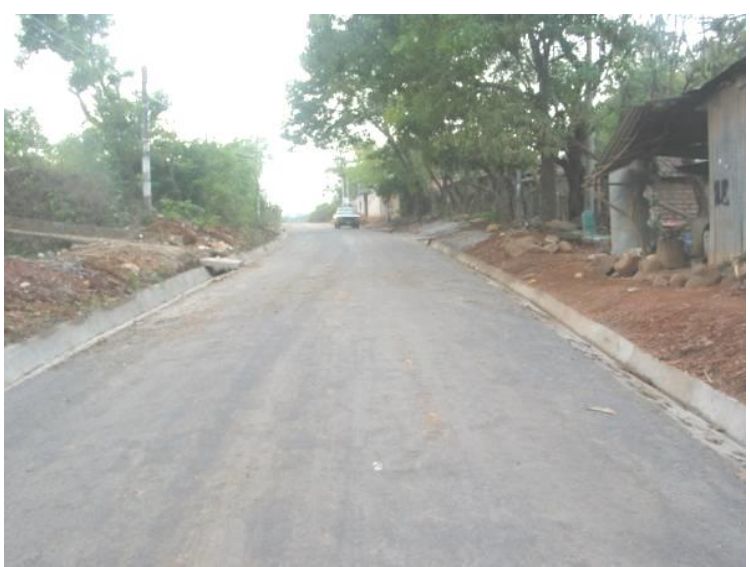

Figura 9. Tramo de vía después de la construcción de un tramo de prueba de estabilización de suelo con características plásticas.

Es de importancia mencionar, que la realización de este tipo de actividad proporciona al profesional un beneficio adicional (la satisfacción personal), ya que además de poner en práctica los conocimientos adquiridos y comprobar el buen desempeño de la obra realizada, implícitamente contribuye a la conservación del medio ambiente y a mejorar las condiciones de vida de los habitantes de la zona intervenida, que son personas de bajos recursos. Figuras 10 y 11.

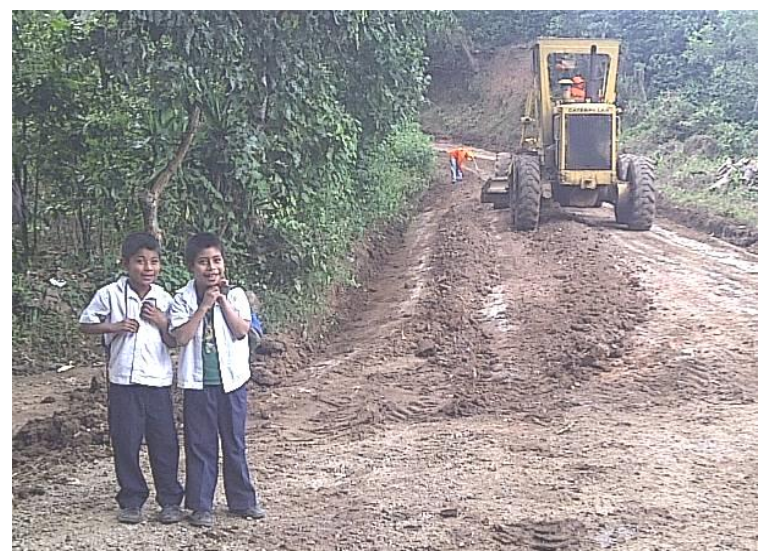

Figura 10. Niños beneficiados con la construcción de tramos de prueba, realizados sobre la ruta que conduce a su escuela.

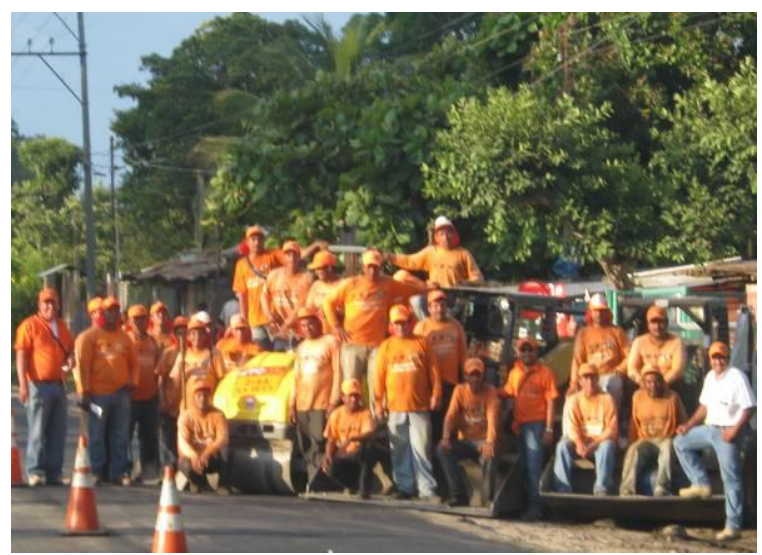

Figura 11. Personal de empresas contratistas y supervisoras, satisfechos de haber realizado la construcción de un tramo de prueba.

\footnotetext{
(3) PUAD consiste en la construcción de una estructura de pavimento formada por el suelo existente en el camino, mezclado con un porcentaje de cemento obtenido según diseño de mezcla (usualmente entre $10 \%$ y $20 \%$ en peso), que forma una sola capa compactada diseñada para soportar las cargas y el desgaste producido por el tráfico vehicular.
} 
Revista ALCONPAT, Volumen 2, Número 1, Enero-Abril 2012, Páginas 29 - 36

\subsection{Evaluación del programa de enseñanza- aprendizaje.}

La evaluación de este programa permite evidenciar si la intervención llevada a cabo es correcta y eficaz. Por tanto, dicha evaluación no es un acto puntual, sino que sirve para una mejora continuada del programa, entendida como un proceso en constante retroalimentación.

La evaluación de la efectividad del proceso de enseñanza aprendizaje ha sido realizada en forma cualitativa y cuantitativa, la cual se describen a continuación:

2.3.1 Evaluación Cualitativa: Cambio de actitud. El cambio de actitud de los profesionales que participan en el programa de capacitación de FOVIAL, se ha visto reflejada en una forma muy positiva, tal como se describe a continuación:

- El profesional participa activamente en el proceso de capacitación, solicita ser invitado a estas capacitaciones y sugiere temas de interés. Existe un mayor acercamiento entre el profesional y personal al interior de FOVIAL, a fin de dilucidar dudas sobre aspectos técnicos.

- A la fecha, ya existen varias experiencias en las que el profesional ha realizado una réplica de los conocimientos adquiridos, proponiendo otras alternativas a las tradicionalmente utilizadas, construye tramos de prueba en sus respectivos proyectos e invita a que se le de acompañamiento en el desarrollo de estos.

- Existe un mayor acercamiento por parte de entidades expertas en temas relacionados al mantenimiento vial, con el objetivo de colaborar en el tema de capacitación, al grado que FOVIAL ya ha firmado convenios con algunas de estas entidades.

- La información de control de calidad generada en los proyectos, presentada al FOVIAL, ha mejorado sustancialmente en contenido y forma.

- Existe un mayor interés, por parte de las empresas contratistas y supervisoras, en el tema de la cultura de calidad. Se evidencia un mayor interés en mantener un nivel de efectividad de sus procesos constructivos, llevando un detallado análisis estadístico de los mismos.

2.3.2 Evaluación cuantitativa: Cambio de aptitud. En referencia al cambio de aptitud del profesional, esta se refleja en la efectividad de los procesos constructivos realizados. Para realizar dicha cuantificación se continúo con el registro de No Conformidades durante el periodo comprendido entre los años 2009 al 2011.

A continuación se describen algunos datos de interés correspondientes al análisis estadístico de No Conformidades, llevado a cabo en el año 2011:

- Las No Conformidades impuestas en el año 2011 fue de 120 (promedio de 40 No conformidades por trimestre), siendo esta cantidad inferior a las 63 No conformidades impuestas en el trimestre comprendido entre mayo a julio de 2008.

- En el análisis estadístico realizado a la obra ejecutada en vías pavimentadas en el año 2011, se observó que de un total de 11,819 muestras de obra ensayada, únicamente 80 fueron objeto de No Conformidad, lo cual representa el 0.69\% de obra No Conforme. Figura 12.

- En el análisis estadístico realizado a la obra ejecutada en vías no pavimentadas en el año 2011, se evidencio que de un total de 8,255 muestras de obra ensayada, solamente 40 fueron objeto de No Conformidad, lo cual representa el $0.49 \%$ de obra No Conforme. Figura 13.

- En ambos gráficos se observa que el porcentaje de producto No Conforme es poco significativo en relación al tamaño del lote muestreado. 
GRAFICO DE ESTATUS DE OBRA REALIZADA DURANTE EL MANTENIMIIENTO DE VIAS PAVIMENTADAS. AÑo 2011

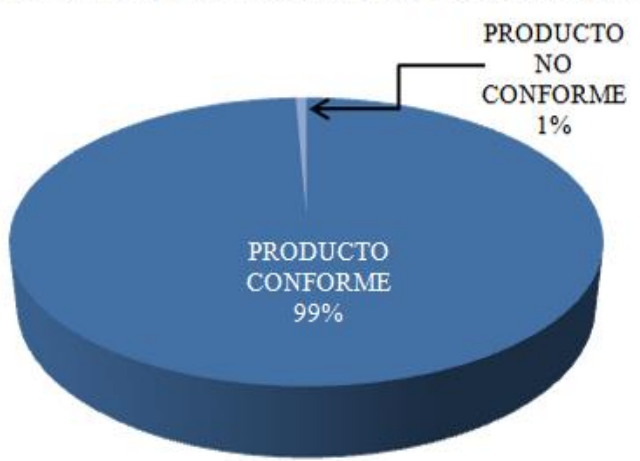

Figura 12. Representación gráfica del análisis estadístico de producto No Conforme en el mantenimiento de vías pavimentadas, llevado a cabo durante el año 2011.

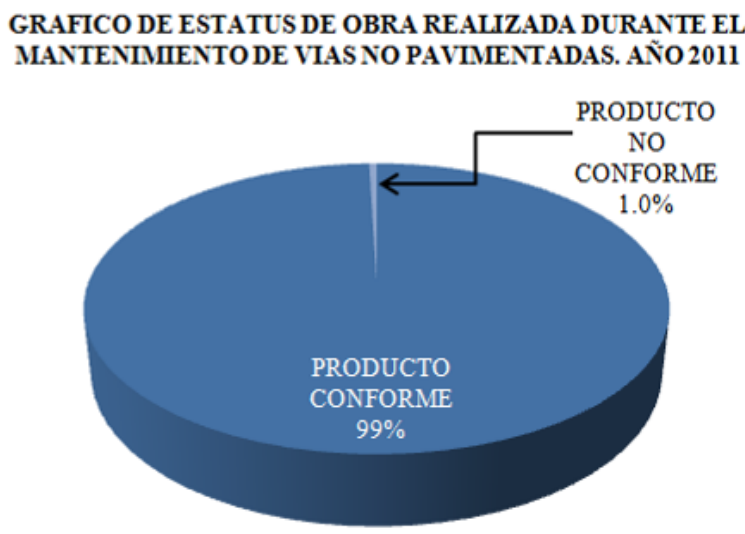

Figura 13. Representación gráfica del análisis estadístico de producto No Conforme en el mantenimiento de vías no pavimentadas, llevado a cabo durante el año 2011.

\section{CONCLUSIONES.}

- El proceso de capacitación de los profesionales ha sido determinante en la mejora de los procesos constructivos realizados, y por consiguiente, en la calidad de la obra construida.

- La adquisición de conocimientos sobre diferentes técnicas constructivas, ha permitido al profesional optimizar los recursos y brindar un mejor aporte al mantenimiento de la red vial de El Salvador.

- El programa de capacitación del FOVIAL, además de proporcionar las herramientas básicas para un efectivo y eficaz control de calidad de las obras, ha logrado mejorar la interrelación entre todas las partes involucradas en cada una de las obras que se ejecutan.

\section{AGRADECIMIENTOS}

El Fondo de Conservación Vial de El Salvador (FOVIAL) agradece el apoyo técnico, logístico y donaciones recibidas de HORCALSA (Cementos Progreso de Guatemala), Asfaltos de Centroamérica S.A de C.V. (ASFALCA), HOLCIM e Instituto Salvadoreño del Cemento y el Concreto (ISCYC), empresas contratistas y supervisoras.

\section{REFERENCIAS}

ISO 9000:2005, Sistema de Gestión de la Calidad. Fundamentos y vocabulario. ISO 9001:2008, Sistema de Gestión de la Calidad. Requisitos.

ISO 2004:2009, Guía para el Éxito Sostenido de una Organización: Enfoque de Gestión de la Calidad.

Plan de Control de Calidad del Fondo de Conservación Vial de El Salvador. 